Editorial: Hospital Metropolitano

ISSN (impreso) 1390-2989 - ISSN (electrónico) 2737-6303

Edición: Vol. 28 No 2 (2020) abril-junio

DOl: https://doi.org/10.47464/MetroCiencia/vol28/2/2020/38-47

URL: http://revistametrociencia.com.ec/index.php/revista/article/view/2

Pág: $38-47$

\title{
Mucormicosis rinocerebral: Una revisión bibliográfica a propósito de un caso clínico
}

\section{Rhinocerebral mucormycosis: A literature review and report of clinical case}

\author{
Marco Cedillo Bailón' ${ }^{1 D}$; Andrea Pesántez Quintuña ${ }^{2}$; \\ René Díaz Mora ${ }^{3}$; Fernando Sandoval Portilla ${ }^{4}$ iD \\ Residente de Cirugía Oral y Maxilofacial, Universidad San Francisco de Quito; Quito, Ecuador \\ Residente de Cirugía Oral y Maxilofacial, Universidad San Francisco de Quito; Quito, Ecuador ${ }^{2}$ \\ Departamento de Cirugía Oral y Maxilofacial, Hospital Baca Ortiz; Quito, Ecuador ${ }^{3}$ \\ Departamento de Cirugía Oral y Maxilofacial, Universidad San Francisco de Quito; Quito, Ecuador
}

Recibido: 20/01/2020 Aceptado: 20/03/2020 Públicado: 01/04/2020

RESUMEN

La mucormicosis es una infección fúngica oportunista, poco común causada por hongos del orden de los mucorales. Ocurre a una tasa anual de 1.7 casos por cada millón de personas y presenta una tasa de mortalidad alta que oscila desde el 30 hasta el $90 \%$ de acuerdo con el estado sistémico del paciente. Los escenarios más complejos se observan en pacientes inmunosuprimidos, mientras que, en pacientes competentes, la invasión fúngica es bien controlada por el sistema inmune del huésped. La infección comienza luego de la exposición, inhalación e invasión de esporas dentro de la cavidad oral y nasal desde donde se puede diseminar a otras partes del cuerpo, permitiendo diferentes presentaciones clínicas en pacientes susceptibles. Actualmente, los registros internacionales de mortalidad de mucormicosis en niños con neoplasias van desde 41.3 a 66.6, por lo que el objetivo de este estudio es presentar un caso raro de mucormicosis rinocerebral en un paciente masculino de 4 años quien además presentó como enfermedad base una leucemia linfoblástica aguda B común, tratado con anfotericina B liposomal y debridación quirúrgica de las zonas afectadas. Finalmente, se realizó una revisión sistemática de la literatura disponible con el afán de determinar y describir los signos, síntomas, diagnóstico, tratamiento disponible y pronóstico de esta enfermedad.

Palabras claves: Mucormicosis, leucemia linfoblástica aguda B común, sistema inmune, angioinvasión, anfotericina B liposomal.

\section{ABSTRACT}

Mucormycosis is an uncommon opportunistic fungal infection caused by fungi of the mucoral order. Occurs at an annual rate of 1.7 cases per million people. It has a high mortality rate ranging from 30 to $90 \%$ according to the patient's systemic status. The most complex scenarios are observed in immunosuppressed patients, whereas, in competent patients, the fungal invasion is well controlled by the host's immune system. The infection begins after exposure, inhalation and invasion of spores into the oral and nasal cavity from where it can spread to other parts of the body, allowing different clinical presentations in susceptible patients. Currently, international records of mortality of mucormycosis in children with neoplasms range from 41.3 to 66.6. The objective of this study is to present a rare case of rhinocerebral mucormycosis in a 4-year-old male patient who also presented as a base disease a leukemia acute B-lymphoblastic disease. Our patient was treated with liposomal amphotericin B and surgical debridement of affected areas. In addition, a systematic review of the available literature was carried out with the aim of determining and describing the signs, symptoms, diagnosis, available treatment and prognosis of this disease.

Keywords: Mucormicosis, B-cell Acute Lymphoblastic Leukemia, immune system, angioinvasion, liposomal Amphotericin B.

Marco Andrés Cedillo Bailón: Andrea Pesántez:

Edison René Díaz Mora:

Fernando Sandoval Portilla:
IDs Orcid

https://orcid.org/0000-0002-0110-5731 https://orcid.org/0000-0002-4479-1460 https://orcid.org/0000-0001-9134-8683 https://orcid.org/0000-0003-0600-0936

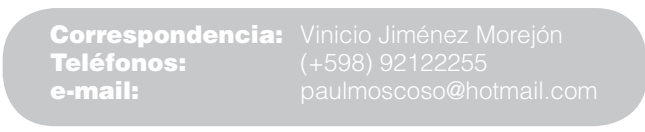




\section{INTRODUCCIÓN}

La mucormicosis es una infección fúngica causada por hongos del orden de los mucorales, fue inicialmente descrita Paltauf en 1885 y actualmente se presenta como la tercera infección fúngica más común en pacientes inmunocomprometidos, se estima que la mucormicosis ocurre a una tasa anual de 1.7 casos por cada millón de personas, presentando un pronóstico pobre, teniendo una tasa de mortalidad desde el 30 al 70\% en infecciones locales y hasta del $90 \%$ en casos diseminados ${ }^{1-4}$.

Aparece como una infección oportunista, poco común, cuyo agente causal se encuentra de manera frecuente en la tierra o en la materia orgánica en descomposición. Los géneros más comúnmente asociados con infecciones humanas son el rhizopus, mucor, rhizomucor, cunninghamella y absidia spp. La invasión de estos hongos no lleva a ningún desenlace clínico importante en pacientes competentes, sin embargo, en pacientes inmunocomprometidos (leucemia, síndrome de inmunodeficiencia, desnutrición, quimioterapia, o quemaduras severas) el desenlace puede ser fatal, llevando a una enfermedad progresiva, favorecida por factores predisponentes, que provocan un curso agudo y una condición nosocomial clásica ${ }^{2-7}$.

La infección comienza luego de la inhalación de esporas dentro de la cavidad oral y nasal desde donde puede diseminarse. Otras rutas de infección incluyen el tracto digestivo y la laceración dérmica, mismas que son las causantes de los diferentes tipos de mucormicosis en diferentes partes del cuerpo. Según su presentación clínica la mucormicosis puede ser clasificada en seis subtipos: rinocerebral, pulmonar, gastrointestinal, cutánea, diseminada, así como otras presentaciones poco frecuentes ${ }^{1,2,5}$.

En el caso de la mucormicosis rinocerebral, Las características clínicas maxilofaciales incluyen edema facial, decoloración cutánea y mucosa, secuestros óseos, fiebre, congestión nasal, rinorrea, dolor sinusal. Como resultado de la extensión retroorbitaria puede aparecer cefalea, ptosis, proptosis, quemosis, conjuntivitis, oftalmoplejía y ceguera; estos hallazgos son importantes, ya que los signos oculares pueden sugerir la extensión cerebral con posterior encefalopatía, cerebritis y trombosis ${ }^{1,3,14}$.

Es importante considerar que las manifestaciones orales y maxilofaciales con frecuencia son los primeros signos clínicos debido a la alta vascularidad de los tejidos blandos orales. Estos signos son importantes ya que el odontólogo puede ser el primero en diagnosticar la enfermedad ${ }^{6,7}$.

El diagnóstico de esta enfermedad depende de una combinación de datos clínicos, hallazgos radiológi- cos y criterio microbiológico. Los tests microbiológicos como una examinación directa son efectivos. Usualmente sustancias aclarantes como el chlorazol negro o dimetilsulfoxido $20 \%$ son usadas y permiten ver bajo el microscopio hifas cenocíticas gruesas, dicotómicas y ramificadas siendo una imagen característica que confirma el diagnóstico ${ }^{7}$.

El cultivo también ha sido empleado como método diagnóstico. El crecimiento ocurre en un lapso de cinco a siete días. Sin embargo, se debe tener en cuenta que estos hongos son contaminantes ambientales y pueden producir resultados falsos positivos, por lo que es importante observar las formas parasitarias de los hongos (hifas cenocíticas) y correlacionarlos con las características clínicas ${ }^{7,12}$.

En cuanto al tratamiento, según la Conferencia Europea de infecciones en Leucemia, el manejo de mucormicosis rinocerebral involucra cuatro áreas: el diagnóstico temprano como primera línea, la terapia antifúngica, la cirugía, y el control de la condición predisponente $e^{5,11}$.

Por su parte, el manejo farmacológico y quirúrgico deben realizarse lo más pronto posible luego del diagnóstico. En el primer caso, el medicamento de elección actualmente es la anfotericina B en formulación lipídica a una dosis de $5 \mathrm{mg} / \mathrm{kg}$ peso. Otras alternativas como el posaconazole y el isovuconazole también han sido presentadas como opciones farmacológicas de tratamiento. Mientras que la extensión del desbridamiento quirúrgico puede variar según la gravedad de la enfermedad ${ }^{14-16}$.

Además, se debe considerar que la mayoría de los pacientes que fallecen con esta enfermedad tienen una función medular pobre y requieren terapia inmunosupresora prolongada. Por lo tanto, no se debe menospreciar todos los esfuerzos terapéuticos para revertir la neutropenia en pacientes hematológicos.

Finalmente, tanto el clínico como el paciente deben comprender que la prevención es importante, particularmente en niños con neoplasias hematológicas bajo tratamiento quimioterapéutico quienes deben tener una excelente higiene oral con un estricto seguimiento y supervisión de los signos y síntomas posibles de esta enfermedad ${ }^{6,16}$.

\section{Objetivos}

Describir un caso raro de mucormicosis rinocerebral en un paciente con enfermedad base de leucemia linfoblástica aguda B común, tratado por el servicio de Cirugía Oral y Maxilofacial del Hospital de Especialidades Pediátricas Baca Ortiz.

Establecer un protocolo de manejo y tratamiento basado en evidencia científica. 


\section{Caso clínico}

Se presenta el caso de un paciente masculino de 4 años con antecedente de leucemia linfoblástica aguda B común diagnosticado desde el 2016 quien presenta recaída medular en el 2019, Ilega al servicio de Cirugía Oral y Maxilofacial del Hospital de Especialidades Pediátricas Baca Ortiz, luego de haber sido diagnosticado y tratado por el servicio de oncohematología.

Somos interconsultados por un cuadro infeccioso agudo con evolución desde el 20 de febrero de 2019. A la evaluación clínica, se examina a un paciente en cama, con fascie álgica y presencia de celulitis facial en tercio medio derecho, de menos de 24 horas de evolución, con progresiva y rápida toma sensorial más deterioro neurológico de Glasgow 12/15 y hemiplejia izquierda. El paciente además presentaba exorbitismo, anisocoria, disminución de reflejo pupilar, parálisis de pares craneales, nistagmos; al examen intraoral se evidencian una importante lesión hemimaxilar derecha de aproximadamente 3 centímetros en región de carrillo derecho y paladar duro. La lesión aparece como una pérdida de sustancia, con coloración alternante entre blanquecina y negruzca, de poca turgencia, eritematosa, dolorosa y de pocos días de evolución.

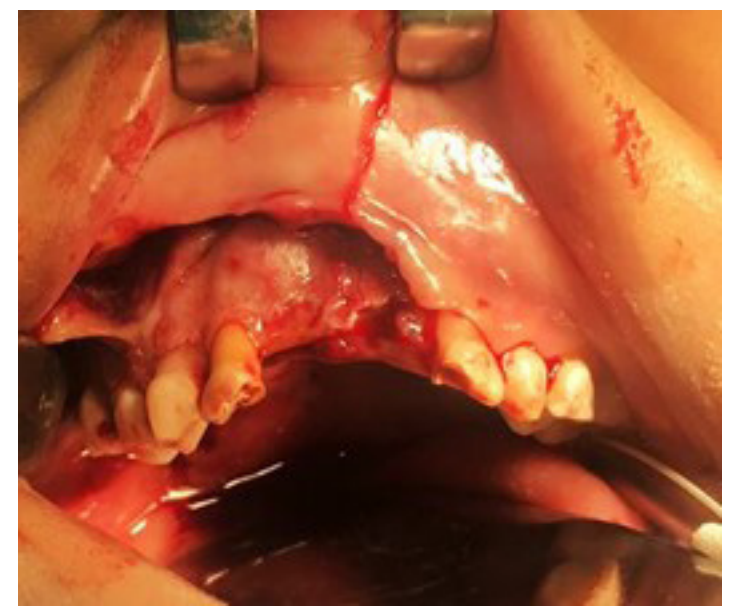

Figura 1. Paciente masculino de 4 años con tejido óseo necrótico en hemimaxilar derecho una vez levantado un colgajo mucoperióstico para exposición de la lesión.

\section{Fuente: Los autores}

Al momento de la evaluación, a la tomografía computarizada se observa sinusitis maxilar y etmoidal con sombras hipodensas en relación con el hueso maxilar del lado derecho. Los resultados de laboratorio del 21/02/2019 demostraron valores de leucocitos 0.1 (5.5 -15.5 mm³), neutrófilos 0.03 (1.50 - 8.50 $\mathrm{mm} * 3)$, linfocitos $0.0(1.5-8.0 \mathrm{~mm} * 3)$, plaquetas de 60.000 (200 - $490 \mathrm{~g} / \mathrm{dL})$, úrea de 2.4 (15.0 - 36.0 $\mathrm{mg} / \mathrm{dL})$, glucosa 249 (60 - $100 \mathrm{mg} / \mathrm{dL})$, creatina de $9.10(0.31-0.47 \mathrm{mg} / \mathrm{dL})$, proteínas $\mathrm{C}$ reactiva de $31(0.00-0.28 \mathrm{mg} / \mathrm{dL})$, procalcitonina 1.3 (0.5 $\mathrm{ng} / \mathrm{mL})$, hematíes 0 (3.5 - $\left.5.3 \mathrm{~mm}{ }^{\star} 3\right)$. El paciente recibe tratamiento antibiótico de amplio espectro a base de amikacina, meropenem, vancomicina, fluconazol y metronidazol. Debido al deterioro general del paciente, con un cuadro clínico compatible con mucormicosis, se decide en conjunto con servicio de oncohematología pediátrica añadir anfotericina B liposomal al esquema, con un esquema de 5 miligramos por kilo de peso, correspondiente en el paciente a 80 miligramos vía endovenosa diluido en 80 mililitros de dextrosa una vez al día. Además, dado el rápido avance y progresivo deterioro de paciente, se decide de manera emergente realizar tratamiento quirúrgico radical de todo el tejido desvitalizado + estudio de cultivo y biopsia + drenaje sinusal maxilar y etmoidal en conjunto con servicio de otorrinolaringología. El procedimiento se realiza el 01/03/2019 y concluye sin complicaciones, posterior a lo cual el paciente es trasladado a la unidad de cuidados intensivos donde permanece orointubado bajo sedoanalgesia y relajación, siendo mantenido en observación y bajo espectro de anfotericina B liposoma a $5 \mathrm{mg} / \mathrm{kg}$ de peso. En el transcurso de su evolución el día 08/03/2019 el paciente presenta parada cardiorrespiratoria que requiere reanimación, con resultado a los 3 minutos, sin embargo, en la evaluación posterior, se valora pupilas midriáticas fijas y reflejo corneal ausente. El paciente permanece en estado crítico y es valorado por neurología quienes inmediatamente solicitan tomografía de control donde se observa hemorragia intraventricular por lo que se sugiere drenaje siempre y cuando el paciente no presente muerte encefálica. El día 11/03/2019, el paciente permanece en condiciones fisiológicas más de 48 horas, sin respuesta por lo que neurología confirma criterios de muerte encefálica luego de electroencefalografía y se decide limitación de esfuerzo terapéutico. El paciente sufre nueva parada cardiorrespiratoria y es dado por fallecido el mismo día a las 9:30 am.

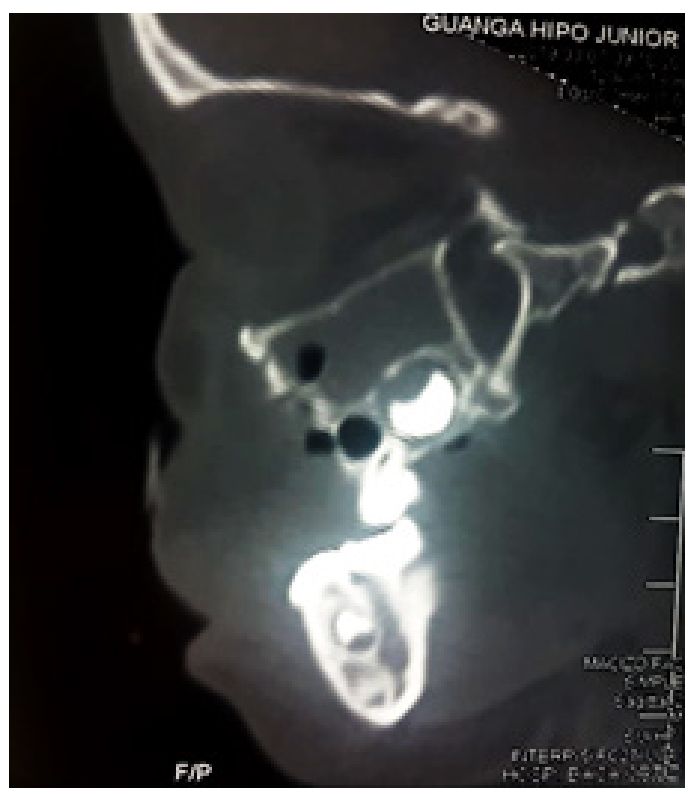




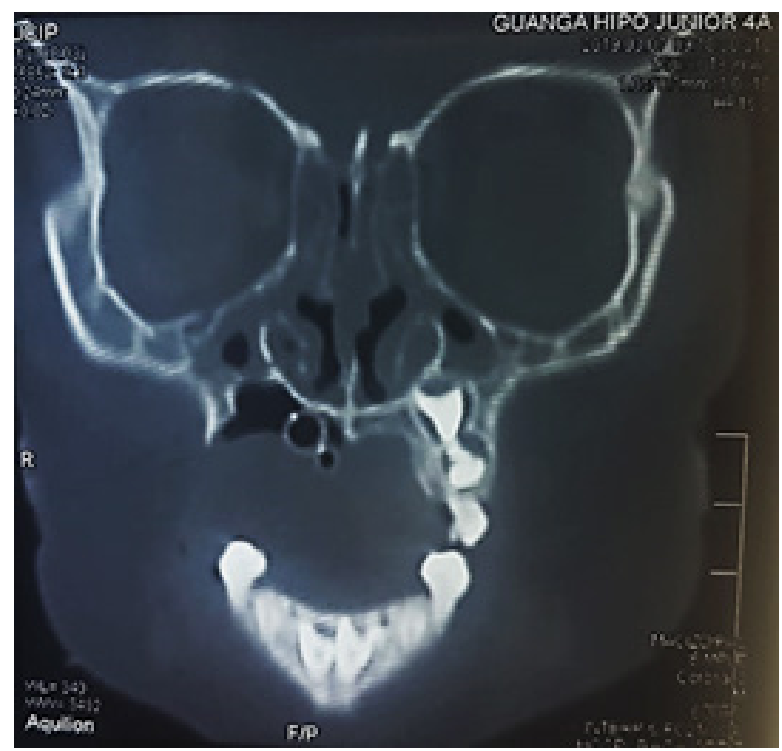

Figura 2. Tomografía computarizada posquirúrgico de la hemimaxilectomía.

Fuente: Los autores

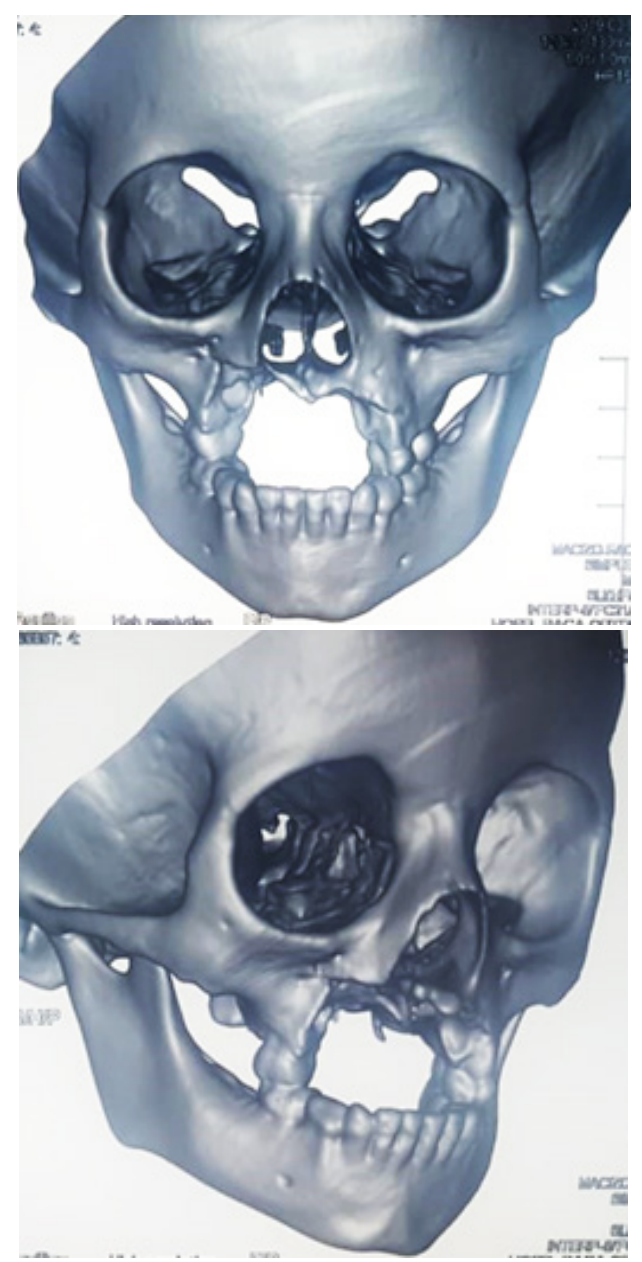

Figura 3. Reconstrucción $3 \mathrm{D}$, posquirúrgico de la hemimaxilectomía.

Fuente: Los autores

\section{DIScusión}

La mucormicosis es una infección fúngica, invasiva y potencialmente fatal, con un rango de mortalidad que oscila entre 20 - 50\% si la infección es localizada y del $70-90 \%$ si es diseminada. Se han reconocido a los agentes causales más comunes como el rhizopus spp con el $48 \%$, y el mucor spp con el $14 \%$. Del grupo rhizopus, el rhizopus arrhizus con el $33 \%$ fue el más común en pacientes con mucormicosis rinocerebral. Otros estudios determinan que el Rhizopus oryzae, es responsable del $70 \%$ de los casos de mucormicosis. Los mucorales también pueden variar según la región geográfica, así por ejemplo el Lichtheimia ha sido el patógeno más común en Europa; y el Atophysomyces fue el menos común, siendo más reportado en regiones tropicales y climas calientes $5,14,16,17$.

Esta enfermedad presenta una rápida evolución y puede llegar a ser altamente mortal sobre todo en pacientes con un sistema inmune deficiente. Varios estudios importantes han corroborado estas estadísticas fatales. La revisión realizada por Roeden sobre 929 casos y el meta-análisis de Vaughan et al. con una revisión de 900 casos han coincidido en sus resultados, donde se pudo concluir que la mayoría de casos se presentó en diabéticos (36\%); en enfermos con neoplasias hematológicas (17\%) y en trasplante de órganos (12\%). Así mismo el meta-análisis de Jeong con 851 pacientes revisados, se analizaron variables estadísticas en cuanto a género, edad, patología base y factores predisponentes; Obteniendo resultados de un rango intercuartílico de edad entre 39 y 61 con una media de 51 años. Además, del total de la muestra estudiada, el 61\% fueron hombres. Por otro lado, la diabetes mellitus fue la causa más común; siendo el factor patológico asociado en el $40 \%$ de los casos, complicándose mucho más con la presencia de cetoacidosis (20\%); las neoplasias hematológicas por su parte representaron el 32\% de los casos mientras que en el 14\%, la mucormicosis se presentó en pacientes con trasplante de órganos. Los factores predisponentes, fue el uso de corticoterapia en el 33\% de los casos, seguido de neutropenia $20 \%$, trauma $20 \%$ y quimioterapia $18 \% 14,17,18$.

El estudio de esta enfermedad fúngica es limitado, con datos epidemiológicos escasos en niños, especialmente en aquellos con enfermedades hematológicas y cáncer. Por lo que en esta revisión se presenta un caso raro de mucormicosis en un paciente masculino de 4 años, quien además presentó como enfermedad base leucemia linfoblástica aguda B común. Actualmente, los registros internacionales de mortalidad de mucormicosis varían desde 41.3 a 66.6 en niños con neoplasias. Siendo este rango compatible con el fatal desenlace en nuestro pacien$t e^{9,12}$. 
Nuestra experiencia guarda correlación con lo que sostiene Muggeo et al; quienes en su estudio con una muestra de 15 pacientes oncológicos pediátricos infectados con mucormicosis pudo observar que la aparición de la enfermedad ocurrió en un tiempo medio de 8,9 meses luego del diagnóstico inicial de la enfermedad predisponente. De esta muestra, el $86,6 \%$ recibieron terapia prolongada con corticoides mientras que el 66,6 \% presentaba neutropenia severa con un tiempo medio de aparición de 42 días y un conteo de neutrófilos menor de $100 \times 10$ 9/L en $46,6 \%$ de los pacientes. Por otro lado, un $33 \%$ de los pacientes tenían diabetes iatrogénica. Muggeo et al resaltan la necesidad de una alteración en una serie de factores sistémicos que comprometen la aparición de la enfermedad ${ }^{9}$.

Es importante indicar que el tratamiento de la leucemia aguda y del linfoma está asociada con una profunda inmunosupresión que puede llevar a una neutropenia severa y prolongada, además, dicho tratamiento, suele requerir el uso prolongado de corticosteroides con un posible desarrollo de diabetes iatrogénica corticoinducida. Estos factores tienen profundos efectos en el sistema inmune permitiendo la supresión de la respuesta de linfocitos T, inhibiendo la producción de interferón y la función fagocíti$\mathrm{ca}^{9}$.

Grossi et al, por ejemplo, indica que las infecciones fúngicas observadas en pacientes con malignidades de las células B tratados con el inhibidor ibrutinib incrementa la susceptibilidad a infecciones fúngicas. Aunque su mecanismo no está totalmente entendido se piensa que el ibrutinib puede inhibir la tirosinquinasa, ya que juegan un papel importante en las defensas contra infecciones fúngicas. Otros estudios multivariados como el realizado por Ming et al también concluyen en que el inicio de las enfermedades micóticas durante la inducción de quimioterapia es significativamente influenciado por tres variables: neutropenia prolongada mayor a 30 días, nutrición parenteral y tabaquismo ${ }^{19,20}$.

Por otro lado, un diagnóstico temprano de mucormicosis no siempre resulta alentador ni garantiza el éxito del tratamiento en todos los casos, ya que como se ha visto, la enfermedad sigue siendo rápidamente progresiva a pesar de los esfuerzos realizados para detener su avance. Sin embargo, estamos de acuerdo que el reconocimiento temprano de esta enfermedad fúngica es crítico y su manejo requiere una cooperación íntima entre cirujanos, hematólogos, infectólogos y microbiólogos, además, una remoción quirúrgica de todo el tejido necrótico resulta indispensable, así como la terapia antifúngica intravenosa, acompañada de control de la enfermedad base. Sin el tratamiento adecuado, la enfermedad es rápidamente mortal.
Complementando lo ya mencionado, en el meta-análisis realizado por Vaughan et al. Se pudo evidenciar que la tasa de supervivencia fue del 59,5\%. El tratamiento temprano se relacionó con una tasa más alta de supervivencia alcanzando hasta el $61 \%$ si se comienza durante los 12 primeros días de presentación. Además, la mortalidad aumentó en casos que no se efectuó una cirugía alcanzando 70\% de mortalidad $^{2,14,15}$

En el inicio de la mucormicosis las esporas diminutas son aerotransportadas y aterrizan en la mucosa oral y nasal donde normalmente los huéspedes inmunológicamente competentes las eliminan por una respuesta fagocítica común. Sin embargo, si esto falla (huésped inmunocomprometido), se producirá la germinación y el desarrollo de hifas. En consecuencia y debido a que los leucocitos polimorfonucleares son menos efectivos para eliminar las hifas, la infección puede establecerse e ir progresando a medida que va invadiendo las arterias. Es ahí desde donde se propagan por las paredes de los vasos y los lúmenes, causando trombosis, isquemia e infarto en los tejidos afectados. La diseminación hematógena puede ocurrir a otros órganos, pudiendo llegar a producir septicemia ${ }^{8}$.

La capacidad de estos hongos para atacar el epitelio previamente dañado permite el desarrollo de la infección. Así, por ejemplo, el Rhizopus invade las paredes endoteliales por reconocimiento especifico de una proteína reguladora de glucosa (GRP78) expuesta luego del daño endotelial. Otras causas de invasión fúngica por trauma se han documentado ya sea por una infección previa, un agente citotóxico o un trauma directo, como por ejemplo una extracción dental; por otro lado, en paciente diabéticos, la concentración de oxígeno, la glucosa alta, el medio ácido y los niveles altos de hierro tienen que ver con la destrucción de células endoteliales en las membranas mucosas favoreciendo también la diseminación de la infección'2,9,10

En personas inmunocompetentes la resistencia antifúngica tiene que ver con la capacidad de restringir la disponibilidad de hierro para el hongo, esto se da mediante la unión del hierro a proteínas como apotransferrina. Este mecanismo es importante ya que, en otras circunstancias, las hifas fúngicas producen una sustancia llamada rizoferrina que permite una rápida unión al hierro formando el complejo hierro-rizoferrina, mismo que es absorbido por el hongo, favoreciendo su vitalidad durante los procesos intracelulares. En consecuencia, el aumento de hierro sérico es otro factor fisiopatológico significante que intensifica la mucormicosis; además de pacientes deprimidos, este escenario también se puede presentar en personas que han sido transfundidas con productos sanguíneos, así como en aquellos tratados con deferoxamine mismo que a pesar de su 
actividad quelante de hierro tiene una afinidad micótica $^{1,21}$.

Otras características propias de los mucorales contribuyen con su naturaleza agresiva. Por ejemplo: la termotolerancia, el crecimiento rápido, la habilidad de obtener hierro del huésped, la regulación negativa de genes implicados en la defensa de huésped, la inhibición en la expresión del interferón y una duplicación de los sistemas implicados en el uso de energía como se revela en el genoma de rhizopus oryzae ${ }^{22}$.

En pacientes diabéticos, el escenario puede volverse aún más favorable al desarrollo de la enfermedad; en estos casos el hongo rhizopus y su enzima cetoreductasa, utiliza los cuerpos cetónicos para prosperar en ambientes acidóticos y altos en glucosa. Además, la acidosis de los pacientes diabéticos altera la función fagocítica de los neutrófilos y contribuye también al aumento de hierro sérico?

Por estas razones, el diagnóstico temprano puede ayudar para la supervivencia del paciente. En general la literatura presenta un tiempo medio de 0 a 30 semanas entre los primeros síntomas y el diagnostico. Dentro de este contexto, la tomografía computarizada y la resonancia magnética son herramientas esenciales al momento de determinar la extensión de la enfermedad ${ }^{12,23}$.

En la tomografía computarizada, la mucormicosis rinocerebral se observa como un ensanchamiento de la mucosa sinusal y en casos más invasivos se puede ver destrucción ósea junto con el edema de los tejidos blandos. En cambio, la resonancia magnética es más sensible en detectar los cambios tempranos de la mucosa infectada y lesionada. Las imágenes van a variar de acuerdo a la cantidad de agua, proteínas y elementos fúngicos presentes. Sin embargo, por lo general permite observar un engrosamiento de la mucosa de la cavidad nasal, obliteración periantral, erosión ósea y orbitaria, compromisos del seno cavernoso y compromiso cerebral. Las lesiones T2 normalmente se presentan como zonas isodensas mientras que en T1 son isointensas en la mayoría de los casos. Mientras que en los casos más severos puede aparecer compromiso cerebral que se hace evidente con áreas hipodensas debido al infarto, producto de la infección ${ }^{23,24}$

Por otra parte, las manifestaciones orales con frecuencia son los primeros signos clínicos debido a la alta vascularidad de los tejidos blandos orales y pueden ocurrir en diferentes niveles de los cuales el más frecuente son las úlceras palatales, siendo considerados en la mayoría de la literatura como los principales signos orales. Se presentan casi siempre úlceras necróticas y delimitadas con bordes bien definidos y pueden ser blancas o negras. Además del paladar, también pueden verse afectadas áreas como el margen alveolar, los labios, las mejillas, la lengua y la mandíbula 1 . En estos casos corresponde realizar un diagnóstico diferencial con la gingivitis ulceronecrotizante, la periodontitis ulceronecrotizante, las ulceraciones traumáticas; el granuloma letal de línea media que es una variación de linfoma, la granulomatosis de Wegener y la sífilis terciaria. Estas 3 últimas tienen una presentación clínica similar que incluye la perforación palatal ${ }^{4-7}$.

La mucosa nasal también se puede ulcerar exponiendo cartílago nasal necrótico, hueso vómer, hueso etmoides y secuestros óseos, formando tejido de granulación y produciendo úlceras palatales que pueden progresar a comunicaciones oroantrales. Desde el paladar y la mucosa nasal, la infección puede progresar hacia la base del cráneo a través de los vasos sanguíneos y diseminarse hacia el sistema nervioso central donde puede involucrar a los nervios craneales II, III, IV y VI deteriorando su funcionalidad. La extensión orbitaria por su lado puede darse a través de los senos etmoidales y maxilares, así como a través del conducto nasolagrimal. Así también la infección cerebral puede ocurrir por diseminación por los vasos orbitarios, agujero óptico y hueso cribiforme, por lo tanto, los signos oculares pueden advertir la posible extensión cerebral ${ }^{10,14,25}$.

Como se dijo anteriormente, existen reportes de mucormicosis derivadas de trauma directo de extracciones dentales, esto se da gracias al sangrado que sirve como una puerta de entrada para infecciones micóticas en regiones maxilofaciales, más en pacientes con factores predisponentes. Estos casos son importantes ya que el odontólogo puede ser el primero en diagnosticar esta enfermedad ${ }^{6,7}$.

El cultivo es otra herramienta útil para confirmar el diagnóstico. Los hongos causantes de la mucormicosis son fácilmente cultivados en Agar Savouraund e incubados a temperatura de 30 a 35 grados centígrados. El crecimiento ocurre de cinco a siete días y sus características histológicas pueden ser verificadas mediante tinciones fúngicas como el Grocott methenamin-sylver ${ }^{1,7}$

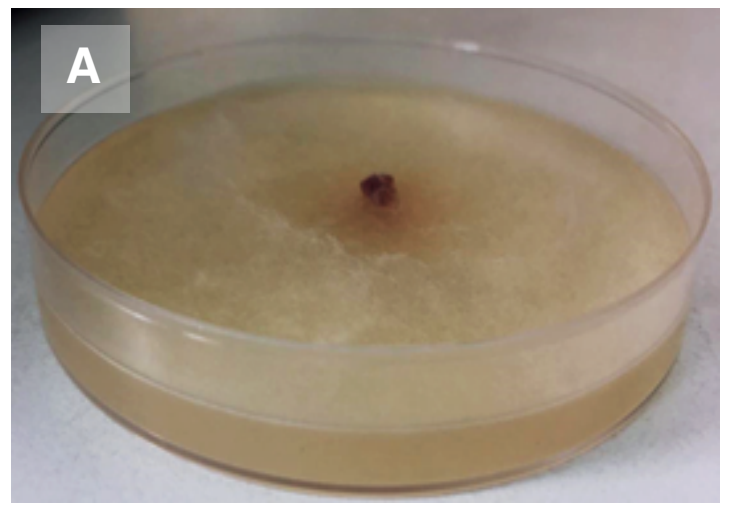



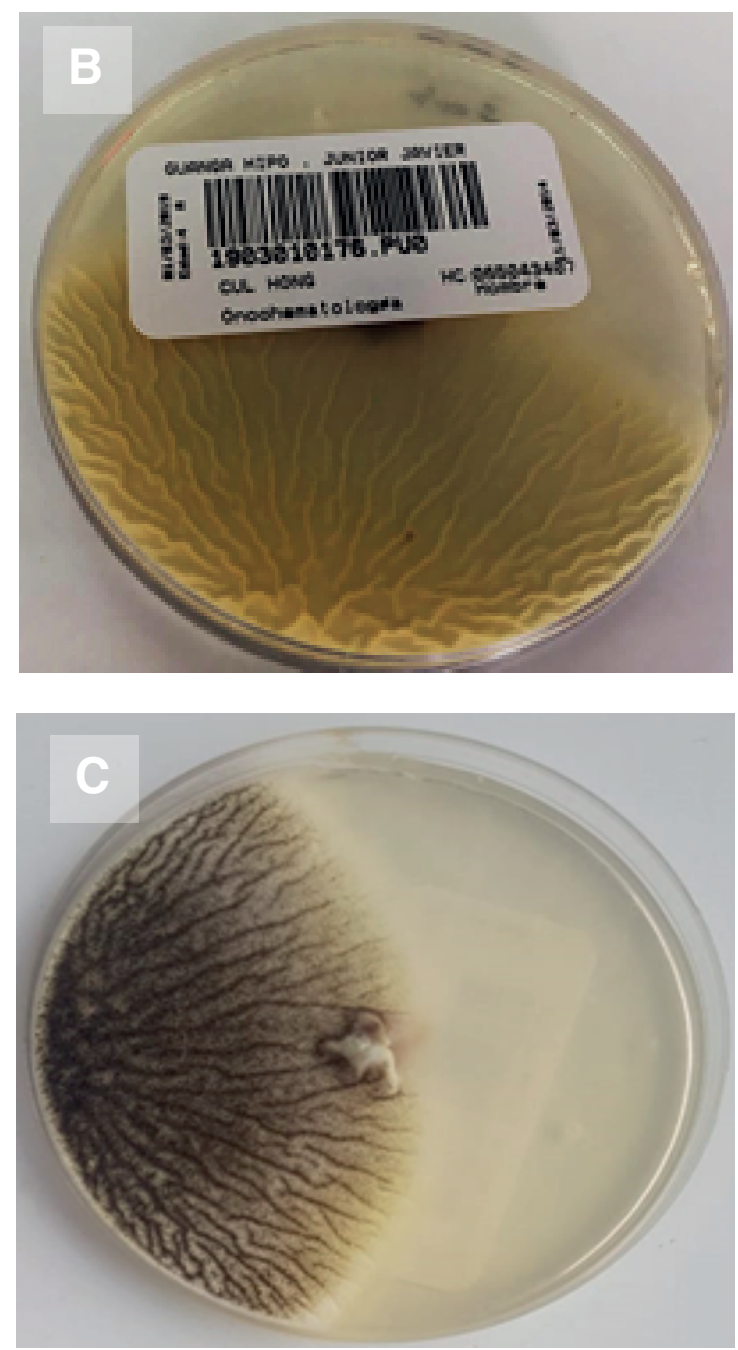

Figura 4. Cultivo de tejido óseo tomado al momento de la hemimaxilectomía del caso clínico presente. (A) etapa de crecimiento inicial. (B) etapa transitoria. (C) última etapa de crecimiento fúngico.

Fuente: Los autores

En el examen de histopatología, se utiliza la tinción de hematoxilina y eosina donde se observan hifas fúngicas anchas, como cintas, irregulares y aseptadas con ramificación en ángulo recto; las cuales se encuentran en su mayoría en áreas adyacentes a las zonas de necrosis especialmente dentro de los vasos necróticos. En este punto, se deben distinguir del Aspergillus, que es más pequeño y septado y con una ramificación más aguda. Por otro lado, el esporangióforo de mucor es más evidente con la tinción de azul de lactofenol de algodón mientras que su angioinvasión se demuestra mediante tinción con hematoxilina y eosina ${ }^{2,8,25}$.

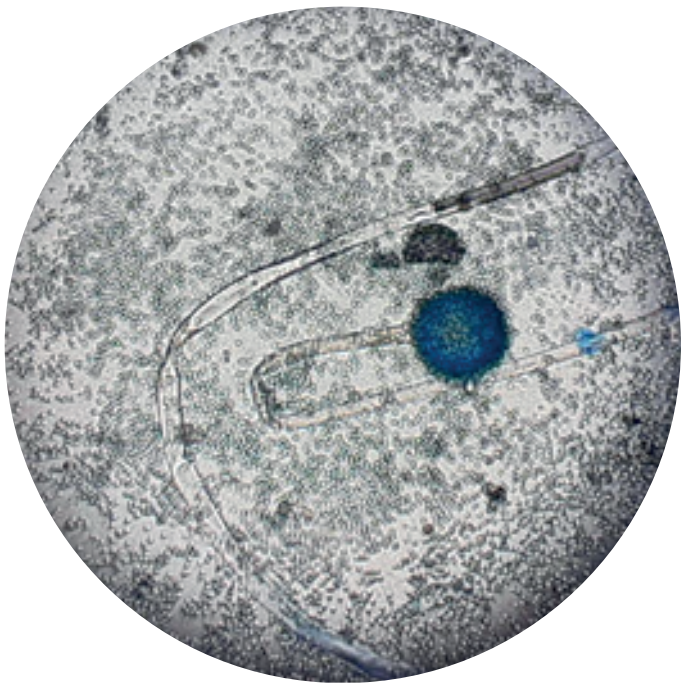

Figura 5. Aspecto microscópico de una de las especies mucor con azul de lactofenol de algodón.

Fuente: Los autores

En los estudios de laboratorio, el principal iniciador de la enfermedad es la neutropenia por lo tanto una biometría hemática resulta importante. Adicionalmente, durante el periodo de tratamiento, una medición de electrolitos y de los niveles de gases en sangre arterial también son de ayuda para detectar tempranamente la acidosis y mantener la homeostasis hasta que se logre recuperar el conteo de células blancas. La monitorización de niveles de hierro también es requerida ya que como se mencionó, los hongos dependen de ellos para favorecer su virulencia. Finalmente, la reacción de la cadena de polimerasa ha aparecido como una nueva herramienta para acelerar el diagnóstico específico para un inicio temprano en el tratamiento antifúngico ${ }^{1,13}$.

Al revisar meta-análisis en búsqueda de obtener conclusiones acerca de la incidencia de presentación, síntomas y pronóstico, los resultados más importantes fueron en el primer caso los obtenidos del meta-análisis realizado por de Jeong, quien demostró que la forma rinoorbitocererbral es la presentación más común con un 30\% seguida de la cutánea con un $22 \%$ y la pulmonar con un $20 \%$. Por su parte, en cuanto a los síntomas y pronóstico de la enfermedad, Casey en su metaanálisis concluyo que los síntomas más comunes fueron el edema periorbitario $27 \%$, fiebre $26 \%$, pérdida de la visión $20 \%$, ptosis $18 \%$, oftalmoplejía 18\% y dolor periorbitario 14\%. La necrosis facial se presentó de manera tardía en solo $12 \%$ de los casos. Mientras que las tasas más bajas de supervivencia se reportaron con necrosis periorbitaria (33\%), trombosis de seno cavernoso (40\%) y edema corneal (40\%). El deterioro neurológico presentó un 34\% de supervivencia, la hemiplejia e infarto un $39 \%$, la necrosis facial un 35\% y la oclusión de la carótida interna un 33\% ${ }^{14,17}$. 
En el tratamiento, se ha establecido a la anfotericina B como la primera línea de agente antifúngico usado contra la mucormicosis. Este fármaco actúa uniéndose a los esteroles formando canales transmembrana que alteran la síntesis de pared celular fúngica. La anfotericina $B$ en su formulación lipídica permite una mejor absorción con menor nefrotoxicidad. Esta está basada en lípidos que aumenta el tiempo de circulación y altera la biodistribución de la anfotericina B asociada. Además, tiene la capacidad de localizar y alcanzar mayores concentraciones en los tejidos infectados e inflamados en comparación con los tejidos normales, que son esencialmente impermeables ${ }^{1,8,14,15}$.

Esta formulación lipídica es usada como tratamiento primario de mucormicosis y su eficacia ha sido probada tanto en laboratorios como en estudios clínicos. Además, tienen un mejor índice terapéutico que la convencional. La dosis optima no está claramente determinada. Sin embargo, la dosis estándar más utilizada es de $5 \mathrm{mg} / \mathrm{kg} /$ día, concordando con lo propuesto por la Conferencia Europea de Infecciones en leucemia con una dosis de 5 a $7,5 \mathrm{mg} / \mathrm{kg}$, aunque se han reportado dosis de hasta $10 \mathrm{mg} / \mathrm{kg}$ de peso $^{9,16}$.

La actividad de la anfotericina B in vitro tiene una alta variabilidad según el tipo de mucorales. Se requiere $2 \mathrm{ug} / \mathrm{ml}$ para l. corymbifela $2 \mathrm{mg} / \mathrm{ml}$ para $\mathrm{m}$. circinelloibes, 4 mg/ml para Arrhizus y 2 ug/ml para Microspores. Dosis más altas de anfotericina liposomal se han asociado con nefrotoxicidad y desordenes electrolíticos $^{16}$.

La administración intravenosa de anfotericina B recomendada es de $5 \mathrm{mg} / \mathrm{kg}$ al día, esta dosis es preparada en una infusión de $1 \mathrm{mg} / \mathrm{ml}$ y administrada a razón de 2,5 mg/kg/hora. Aunque este fármaco tiene una reducción sustancial en cuanto a nefrotoxicidad en relación a la anfotericina $\mathrm{B}$ convencional; un protocolo de administración segura recomendado se mantiene y consiste en inicialmente administrar una dosis intravenosa de $1 \mathrm{mg}$ en $20 \mathrm{ml}$ de dextrosa al $5 \%$, seguido de un control cada 30 minutos de los efectos secundarios como fiebre alta, escalofríos, hipotensión, anorexia, náuseas, vómitos, cefalea, disnea, taquipnea, somnolencia, debilidad generalizada, flebitis, daño renal y anafilaxia hasta completar 2 a 4 horas. Esto debido a que se puede observar una reacción aguda grave de 1 a 3 horas después de la infusión, en tal caso estaría indicado una reducción de la dosis ${ }^{4,8}$.

El tratamiento antifúngico se puede combinar con oxígeno hiperbárico, quelantes de hierro o interferón gamma ${ }^{5}$. Se ha visto que los mucorales son altamente sensibles al oxigeno hiperbárico, ya que además de mejorar el flujo de oxígeno en el tejido isquémico y aliviar la acidosis, al mismo tiempo, el oxígeno hi- perbárico promueve la acción oxidativa de la anfotericina B. La terapia de oxígeno hiperbárico para la mucormicosis debe consistir en la exposición al $100 \%$ de oxígeno durante 90 minutos a 2 horas a presiones de 2.0 a 2.5 atmósferas con 1 o 2 exposiciones diarias para un total de 40 tratamientos ${ }^{2,7,8}$.

La necrosis consecuente es un factor limitante para la terapia antifúngica. Es por eso que la remoción urgente es necesaria. El desbridamiento quirúrgico tiene que ser amplio, involucrando todas las áreas necróticas, incluso puede ser necesarios según los procedimientos para conseguir el control total de la infección ${ }^{15}$.

La cirugía ha demostrado una significativa mejora en la supervivencia comparada con terapia antifúngica solamente. Las imágenes por resonancia magnética determinan la cantidad de resección necesaria. El manejo quirúrgico puede variar. Por ejemplo, un abordaje endoscópico se prefiere cuando el diagnostico ha sido temprano con enfermedad limitada o con comorbilidades sistémicas importantes. La cirugía abierta se prefiere cuando existe enfermedad extensa que incluye maxilectomía o resección craneofacial ${ }^{16}$.

Otros fármacos como los triazoles actúan agotando el ergosterol de la membrana fúngica. Los triazoles más nuevos como isavuconazole y el posaconazole tienen mejor actividad contra mucorales. El isovuconazole, ha sido estudiado como primera línea de terapia de mucormicosis, y el posaconazole, como una terapia secundaria; Ambos han sido presentados como opciones alternativas de tratamiento. No obstante, la eficacia de estos agentes se basa en datos clínicos limitados y en datos preclínicos in vivo e in vitro que muestran actividad contra mucorales ${ }^{14,16}$.

El isavuconazole es un triazol nuevo de amplio espectro, se lo usa cuando la anfotericina B no está disponible, este viene en presentaciones intravenosas y orales y se administra con una dosis de carga 200mg cada 8 horas por 2 días continuada por una dosis 200mg al día. Presenta menos interacciones medicamentosas, menos toxicidad, menos efectos secundarios en piel y ojos y excelente viabilidad sin requerimientos de comida. Los valores de concentración mínima inhibitoria de isavuconazole es de dos a cuatro veces más alta comparada con posaconazole. Se lo debe utilizar como terapia de reemplazo en caso de pacientes severamente comprometidos luego de la falla del posaconazole. El rango de la concentración mínima inhibitoria varía entre 0.25 a $16 \mathrm{ug} / \mathrm{ml}^{16}$

El posaconazole tiene una activada variable in vitro dependiente de la especie de mucorales; el coeficiente mínimo inhibitorio va a variar ampliamente de 
1.0 a $8.0 \mathrm{ug} / \mathrm{ml}$. Puede ser una segunda alternativa de tratamiento en pacientes que no responde o no toleran la anfotericina B liposomal, es bien tolerada y presenta solo unos pocos efectos colaterales gastrointestinales ${ }^{16}$.

El voriconazol es un nuevo derivado de triazol con actividad in vitro e in vivo contra una amplia gama de patógenos fúngicos. Tiene superioridad sobre la anfotericina B por su capacidad para penetrar en el sistema nervioso central. Sin embargo, los efectos secundarios incluyen trastornos visuales transitorios, fiebre, erupción cutánea, vómitos, náuseas, diarrea, cefalea, sepsis, edema periférico, dolor abdominal y trastorno respiratorio ${ }^{4}$.

La combinación de antifúngicos se ha convertido en una práctica bastante frecuente en búsqueda de efecto sinérgico, con espectro más amplio. En estudios in vitro e in vivo existe evidencia del sinergismo entre polienos y equinocandinas. Principalmente en pacientes con mucormicosis rinocerebral favorecido por un cuadro de cetoacidosis. Sin embargo, en casos de pacientes con neoplasias hematológicas esta combinación no fue satisfactoria. La combinación de anfotericina B más triazol para el tratamiento de mucormicosis muestra evidencia contradictoria ya que mientras que los estudios in vitro muestran sinergismo, otros estudios in vivo realizados en modelos roedores no mostraron ningún beneficio cuando se usaron de manera concomitante. Finalmente, la modesta evidencia actual con datos clínicos y preclínicos no respalda el uso de terapia combinada ${ }^{16}$.

Algunas consideraciones para prevenir la aparición de mucormicosis están recomendadas en la literatura, tal es el caso de la prevención o la reducción del tiempo de neutropenia para lo cual el tratamiento con factores de crecimiento y la trasfusión de células blancas puede ser considerada; además la corrección de la hiperglicemia y la acidosis también debe ser llevada a cabo, así como reducir las concentraciones de hierro mediante el uso de quelantes, esto debido a que el aumento de hierro sérico intensifica la mucormicosis. La prevención de la trombocitopenia tampoco se debe pasar por alto, ya que las plaquetas Ilevan importantes inmunoreguladores y propiedades antifúngicas. Por último, se debe recalcar que los niños con neoplasias hematológicas bajo tratamiento quimioterapia deben tener una excelente higiene oral llevada bajo supervisión paterna6,22.

El incremento de la presión de oxígeno logrado con la terapia de oxígeno hiperbárico mejora la funcionalidad de los neutrófilos y promueve la función de la anfotericina B reduciendo la acidosis además inhibe el crecimiento fúngico y mejora las tasas de cicatrización. En una revisión de 28 casos el oxígeno hiperbárico fue beneficioso en paciente diabéticas (supervivencia del 94\%) pero no en pacientes con neoplasias hematológicas o trasplante medular (supervivencia 33\%, p 0.02)

Otras estrategias que favorezcan el sistema inmune como la administración de granulocitos, factores estimulantes de colonias o interferón gamma han sido propuestas como terapia adjunta. La trasfusión de granulocitos ha sido probada con resultados controversiales, pero con riesgo de lesión pulmonar; el interferón gamma restaura la función de los monocitos y han sido probados como terapia de rescate en infecciones fúngicas con compromiso de vida ${ }^{16}$.

El calcineriun es una proteína fosfatasa con rol importante en los procesos fisiológicos fúngicos útiles para promover la resistencia contra los antifúngicos; la terapia con inhibidores del calcineriun tales como el tacrolimus, sirolimus, y la ciclosporina A son comúnmente administrados. Como agentes inmunosupresores que también presentan actividad antifúngica además que presenta sinergismo con la terapia antifúngica ${ }^{22}$

Estudios futuros deberían explorar el uso de inhibidores de calcineriun como una parte integral del tratamiento. Otros tipos de drogas con posibles propiedades antifúngicas son las estatinas. En varios estudios se han visto el efecto antifúngico sinérgico de la lovastatina con azoles in vitro, además que un estudio experimental encontró que el uso de estatinas reduce significativamente varias funciones biológicas del rhizopus oryzae ${ }^{22}$.

Actualmente no existe duración estándar para el tratamiento de la mucormicosis. Se debe valorar cada caso de manera individual y todo tratamiento antifúngico se debe continuar hasta la total resolución de todos los signos y síntomas de la infección y el control de la inmunosupresión. En ciertos pacientes la tomografía computarizada con emisión de positrones puede ayudar haciendo una distinción entre los signos radiográficos de enfermedad activa e inactiva $^{16}$.

\section{CONCLUSIONES Y RECOMENDACIONES}

Con el objetivo de un correcto manejo del paciente con mucormicosis, se concluyen los siguientes puntos:

- La mucormicosis es una enfermedad altamente letal, que presenta predisposición por pacientes inmunocomprometidos.

- La clínica sugestiva debe ser confirmada mediante la obtención de cultivos y biopsias de las zonas afectadas, esto permitirá una mejor oportunidad de éxito gracias al diagnóstico temprano.

- La detección y el tratamiento oportuno con tera- 
pia de combinación dan mejores resultados en comparación con la monoterapia.

- Según la literatura, un control local se obtuvo en un $90 \%$ de los pacientes en cirugía radical vs en $46 \%$ en cirugía limitada.

- Existen algunas alternativas terapéuticas. Sin embargo, el control del estado sistémico del paciente y el mantenimiento de las condiciones fisiológicas juegan un rol trascendental para la supervivencia del paciente.

\section{CONTRIBUCIÓN DE LOS AUTORES}

Marco Cedillo conceptualizó y diseñó los datos, los recopiló, analizó e interpretó los datos, redactó el artículo y revisó críticamente el artículo.

Andrea Pesántez conceptualizó y diseñó los datos, los recopiló, analizó e interpretó los datos, redactó el artículo y revisó críticamente el artículo.

René Díaz conceptualizó y diseñó los datos, los recopiló, analizó e interpretó los datos, redactó el artículo y revisó críticamente el artículo.

Fernando Sandoval analizó e interpretó los datos y revisó críticamente el artículo.

\section{CONFLICTO DE INTERÉS}

Los autores declararon no tener ningún conflicto de interés personal, financiero, intelectual, económico y de interés corporativo con el Hospital Metropolitano y los miembros de la revista MetroCiencia.

\section{REFERENCIAS BIBLIOGRÁFICAS}

1. Cohen A, Shoukair F, Koren M, Shaulov A \& Casap N. Successfu Mandibular Mucormycosis Treatment in the Severe Neutropenic Patient. Journal of Oral and Maxillofacial Surgery; 2019. https://doi.org/10.1016/j. joms.2019.02.012

2. Singh VP, Bansal C, Kaintura M. Sinonasal Mucormycosis: A to Z. Indian J Otolaryngol Head Neck Surg. 2019;71(Suppl 3):1962-1971. https://doi. org/10.1007/s12070-018-1384-6

3. Jundt JS, Wong MEK, Tatara AM, Demian NM. Invasive Cutaneous Facial Mucormycosis in a Trauma Patient. J Oral Maxillofac Surg. 2018;76(9):1930 e1-1930.e5. https://doi.org/10.1016/j.joms.2018.01.022

4. Singh V, Sharma B, Sen R, Agrawal S, Bhagol A, \& Bali R. Rhinocerebra Mucormycosis: A Diagnostic Challenge and Therapeutic Dilemma in Immunocompetent Host. Journal of Oral and Maxillofacial Surgery; 2012;70(6) 1369-1375. https://doi.org/10.1016/j.joms.2011.06.209

5. Pajpani $\mathbf{M}$, \& Webb R. Lingual necrosis caused by mucormycosis in a patient with aplastic anaemia: case report. British Journal of Oral and Maxillofacial Surgery; 2014; 52(10), e144-e146. https://doi.org/10.1016/j. bjoms.2014.09.012

6. Dogan C, Leblebisatan G, Haytac, C, Antmen B, \& Surmegozler O. Ora mucormycosis in children with leukemia: report of 2 cases. Quintessence international; 2007; 38 6, 515-20. Disponible en: https://pubmed.ncbi.nlm. nih.gov/17625636/

7. Bonifaz A, Macias B, Paredes-Farrera F, Arias P, Ponce R, \& Araiza J. Palatal zygomycosis: experience of 21 cases. Oral Diseases; 2008;14(6), 569-574. https://doi.org/10.1111/j.1601-0825.2007.01433.x
8. O'Neill M, Alessi S, George B, \& Piro J. Disseminated Rhinocerebral Mucormycosis: A Case Report and Review of the Literature. Journal of Oral and Maxillofacial Surgery; 2006; 64(2), 326-333. https://doi.org/10.1016/j. joms.2005.10.017

9. Muggeo P, Calore E, Decembrino N, et al. Invasive mucormycosis in children with cancer: A retrospective study from the Infection Working Group of Italian Pediatric Hematology Oncology Association. Mycoses. 2019;62(2):165-170. https://doi.org/10.1111/myc.12862

10. Soni A. Paranasal Mucormycosis in an Immunocompetent Individual: Importance of Early Diagnosis. Int J Oral Health Med Res 2017;4(3):52-56.

11. Klimko N, Khostelidi S, Shadrivova O, Volkova A, Popova M, Uspenskaya O, Afanasyev B. Contrasts between mucormycosis and aspergillosis in oncohematological patients. Medical Mycology; 2019; 57(Supplement_2), S138-S144.

12. Cornu M, Bruno B, Loridant S, Navarin P, François $\mathbf{N}$, Lanternier F, Sendid B. Successful outcome of disseminated mucormycosis in a 3-year-old child suffering from acute leukaemia: the role of isavuconazole? A case report. BMC Pharmacology and Toxicology; 2018; 19(1). https://dx.doi. org/10.1186/s40360-018-0273-7

13. Bellanger P, Berceanu A, Rocchi S, Valot B, Fontan J, Chauchet A, Millon L. Development of a quantitative PCR detecting Cunninghamella bertholletiae to help in diagnosing this rare and aggressive mucormycosis. Bone Marrow Transplantation; 2018. https://doi.org/10.1038/s41409-018-0194-5

14. Vaughan $\mathbf{C}$, Bartolo A, Vallabh $\mathbf{N}$, \& Leong C. A meta-analysis of survival factors in rhino-orbital-cerebral mucormycosis-has anything changed in the past 20 years? Clinical Otolaryngology; 2018. https://doi.org/10.1111/ coa. 13175

15. Debureaux PE, Paccoud O, Guitard J, et al. Rhino-orbital Mucormycosis presenting as facial cellulitis in a patient with high-risk acute myeloid leukemia in relapse. Curr Res Transl Med. 2019;67(2):76-78. https://doi. org/10.1016/j.retram.2019.01.004

16. Sipsas NV, Gamaletsou MN, Anastasopoulou A, Kontoyiannis DP. Therapy of Mucormycosis. J Fungi (Basel). 2018 Jul 31;4(3):90. https://doi. org/10.3390/jof4030090

17. Jeong W, Keighley C, Wolfe R, et al. The epidemiology and clinical manifestations of mucormycosis: a systematic review and meta-analysis of case reports. Clin Microbiol Infect. 2019;25(1):26-34. https://doi.org/10.1016/j. cmi.2018.07.011

18. Roden M, Zaoutis E, Buchanan L, Knudsen A, Sarkisova A, Schaufele L, Walsh J. Epidemiology and Outcome of Zygomycosis: A Review of 929 Reported Cases. Clinical Infectious Diseases; 2005; 41(5), 634-653. https:// doi.org/10.1086/432579

19. Grossi O, Pineau S, Sadot-Lebouvier S, Hay B, Delaunay J, Miailhe F, Le Pape P. Disseminated mucormycosis due to Lichtheimia corymbifera during ibrutinib treatment for relapsed chronic lymphocytic leukaemia: a case report. Clinical Microbiology and Infection; 2018. https://doi.org/10.1016/i. cmi.2018.10.004

20. Lien Y, Chou H, Lin C, Bai Y, Chiu F, Yeh P. Epidemiology and risk factors for invasive fungal infections during induction chemotherapy for newly diagnosed acute myeloid leukemia: A retrospective cohort study; 2018; 13(6): e0197851. https://doi.org/10.1371/journal.pone.0197851

21. Cheema SA, Amin F. Five cases of rhinocerebral mucormycosis. $\mathrm{Br}$ J Oral Maxillofac Surg. 2007;45(2):161-162. https://doi.org/10.1016/j. bjoms.2005.06.021

22. Petrikkos G, \& Tsioutis C. Recent Advances in the Pathogenesis of Mucormycoses. Clinical Therapeutics; 2018; 40(6), 894-902. https://doi.org/10.1016/j.clinthera.2018.03.009

23. Ergun O, Tahir E, Kuscu O, Ozgen B, \& Yilmaz T. Acute Invasive Fungal Rhinosinusitis: Presentation of 19 Cases, Review of the Literature, and a New Classification System. Journal of Oral and Maxillofacial Surgery; 2017; 75(4), 767.e1-767.e9. https://doi.org/10.1016/j.joms.2016.11.004

24. Klein-Awarjanow K, Nowicki K, Bieniaszewska M, \& Szurowska E. Common radiological findings in fungal infections in hematological patients - Review. Polish Annals of Medicine; 2017; 24(2), 287-294. https://doi.org/10.1016/j.poamed.2016.12.001

25. Marx R \& Stern D. Oral and Maxillofacial Pathology: a rationale for diagnosis and treatment. Second edition; 2012; 110 - 112

Como citar este artículo: Cedillo M, Pesántez A, Díaz R, Sandoval F. Mucormicosis rinocerebral: Una revisión bibliográfica a propósito de un caso clínico. MetroCiencia. 2020 abr 1;28(2):38-47 https://doi.org/10.47464/MetroCiencia/vol28/2/2020/38-47 